\title{
DETERMINACIÓN DE LOS ESFUERZOS DE DISEÑO DE VIGAS \\ LAMINADAS DE PINO CARIBE (Pinus caribaea var. hondurensis) ENCOLADAS CON ADHESIVO DE ISOCIANATO (MDI).
}

\section{DETERMINATION OF STRESS PARAMETERS OF GLUED-LAMINATED TIMBER OF CARIBEAN PINE (Pinus caribaea var. hondurensis) GLUED WITH ISOCYANATE-BASED ADHESIVES (MDI).}

\author{
Wilver Contreras M. ${ }^{1}$, Styles Will Valero ${ }^{2}$, Edward Thomson ${ }^{3}$, Mary Elena Owen de C. ${ }^{4}$, Eric Barrios ${ }^{5}$.
}

\section{RESUMEN}

A través del Laboratorio Nacional de Productos Forestales de la Universidad de Los Andes (Mérida, Venezuela) se realizaron los ensayos de flexión estática, según la Norma ASTM D-198-84, para la determinación de los esfuerzos de diseño de 18 vigas de madera laminada de pino caribe encoladas con adhesivo de isocianato (MDI). Las mismas fueron fabricadas íntegramente en la única industria de madera laminada existente, hasta el presente, en Venezuela. Estas vigas laminadas se dividieron en dos tipos, 9 estaban constituidas por láminas completas de madera y las otras 9 estaban constituidas por láminas de madera seccionadas y unidas por medio de la técnica del finger joint; dentro de cada uno de estos tipos se prepararon tres vigas laminadas con anchos distintos ( $65 \mathrm{~mm}, 95 \mathrm{~mm}$ y $125 \mathrm{~mm})$, siendo sus alturas y sus largos iguales ( $270 \mathrm{~mm}$ y $3500 \mathrm{~mm}$ respectivamente). Al comparar los resultados obtenidos con valores de vigas laminadas de pino radiata de Chile, y pino resinoso de Argentina se logró determinar, que los valores de Esfuerzo Límite Proporcional, Módulo de Ruptura y Módulo de Elasticidad para todas las vigas estudiadas, estaban por debajo. El análisis morfológico de todas las vigas, definió que éstas presentaron grandes deficiencias en la calidad física de la madera y las líneas de cola, y por igual, en el proceso de fabricación. Estos factores, son la principal causa de los bajos valores obtenidos. Se recomienda, en esta primera etapa de la industria, mejorar los aspectos técnicos señalados, para aumentar la resistencia y calidad estructural de las vigas laminadas encoladas.

Palabras claves: Vigas laminadas encoladas, pino caribe, ensayos, esfuerzos de diseño, ensayos de flexión.

\footnotetext{
${ }^{1}$ Dr. Arq. MSc. Centro de Estudios Forestales y Ambientales de Postgrado (CEFAP - ULA). Facultad de Ciencias Forestales y Ambientales. Laboratorio Nacional de Productos Forestales (LNPF). Universidad de Los Andes, Mérida, Venezuela. wilconmi@doctor.upv.es

${ }^{2}$ Ing. For. MSc. Laboratorio Nacional de Productos Forestales (LNPF). Universidad de Los Andes, Mérida, Venezuela. styles@ula.ve

${ }^{3}$ Dr. Ing. Postgrado de Cálculo Estructural. Facultad de Ingeniería. Universidad de Los Andes. Mérida. Venezuela.

${ }^{4}$ Dra. Arq. MSc. Escuela de Diseño Industrial (EDI) de la Facultad de Arquitectura y Arte. Investigadora del Laboratorio Nacional de Productos Forestales (LNPF). Universidad de Los Andes, Mérida, Venezuela. marowde@ doctor.upv.es

${ }^{5}$ Ing. Ind. For. M.Sc. Estudiante Doctorado de Arquitectura y Urbanismo de la UCV. Profesor de la Universidad Nacional Experimental de Guayana (UNEG). Investigador del Centro Biotecnológico de Guayana (CEBIOTEG). Upata, Edo. Bolívar. Puerto Ordaz.Venezuela. ericbarrios@latinmail.com

Autor para correspondencia: wilvercontrerasmiranda@yahoo.es

Recibido: 09.05. 2007. Aceptado: 26.07. 2007.
} 


\begin{abstract}
Static flexion tests of eighteen glued-laminated timber of caribean pine glued with isocyanatebased adhesives (MDI) were carried out following ASTM- D198-84 standards at the National Laboratory of Forest Products of "La Universidad de los Andes" (Mérida, Venezuela). These beams were fabricated, in their entirety, by the only company that develops glued-laminated (GLULAM) timber in Venezuela. The glued-laminated timber was divided into two groups of nine. The first group consisted of GLULAM timber made from whole beams of wood, while the second was composed of sectioned wood that had been finger-joined and later made into GLULAM beams. Each group was divided into three subgroups with glued-laminated timber of widths $65 \mathrm{~mm}, 95 \mathrm{~mm}$ and $125 \mathrm{~mm}$, but with a common height of $270 \mathrm{~mm}$ and a length of $3500 \mathrm{~mm}$. The values obtained for stress at proportional limit, modulus of rupture and the modulus of elasticity for all the beams studied were less than those reported for glued-laminated timber from Chilean radiata pine and Argentinean resinous pine. Morphology studies performed on all the studied beams revealed that they presented great deficiencies in the physical quality of the wood, glue lines, and fabrication process. These factors are responsible for the low-performance of the test samples. It is recommended, in this first stage of the industry, that the mentioned technical aspects be improved so that resistance and structural quality of glued-laminated timber is in turn enhanced.
\end{abstract}

Keywords: Laminated beams, Caribean pine, wood tests, stress parameters, flexion tests.

\title{
INTRODUCCION
}

En la región del oriente de Venezuela, específicamente, al sur de los estados Monagas y Anzoátegui, se encuentra una de las mayores manchas continuas de plantaciones forestales de pino caribe (Pinus caribaea var. hondurensis) del mundo. Este recurso forestal que sobrepasa las 622 mil hectáreas, representa para la actual Administración central del país, la principal fuente de materia prima para el establecimiento, a partir del año 2007, del desarrollo de un programa nacional anual para la construcción de 50 mil viviendas de madera (CVG-Proforca, 2007). Se pretende con este programa, tratar de disminuir el fuerte déficit habitacional nacional que actualmente asciende a los 2 millones de unidades de viviendas (Contreras, 2003). Dentro de los proyectos que está llevando la Corporación Venezolana de Guayana Productos Forestales de Oriente (CVG-Proforca), institución gubernamental rectora del aprovechamiento sostenible de las plantaciones forestales de pino caribe, así como su transformación y comercialización de los productos forestales, es el montaje de una planta de fabricación de productos estructurales de madera laminada encolada (CVG Proforca, 2007).

De ahí, que el estudio continuo de todas las posibilidades técnicas de uso, fortalezas y debilidades que proyecte la madera de pino caribe como materia prima, sea una necesidad urgente y una prioridad a fin de contribuir a solventar, no sólo el déficit habitacional, sino también la fabricación de infinidad de productos forestales para sistemas constructivos, muebles, objetos domésticos, juguetes, etcétera. Por ello, en el contexto en que se desarrolla el presente trabajo, se pretende determinar las verdaderas potencialidades técnicas de esta especie de madera, en lo que se refiere a la fabricación de productos forestales de madera laminada encolada con calidad estructural.

Por lo que este estudio pretende determinar las propiedades mecánicas de las vigas laminadas de pino caribe encoladas con isocianato realizadas por una empresa en este ramo en Venezuela pudiendo, así, evaluar sus características, validar la proyección del uso futuro de las vigas analizadas en los sistemas estructurales de edificaciones y comparar los resultados obtenidos con otros trabajos análogos de madera laminada encolada y de madera aserrada de especies de coníferas en otros países. Para lo cual se consultó la Norma ASTM D-198-84, y a partir de allí se definió toda la estrategia técnica y metodológica a fin de determinar los esfuerzos de diseño de las vigas laminadas de pino caribe. 


\section{MATERIALES Y MÉTODOS}

\section{A. Localización de la industria y características de la materia prima.}

Las vigas de madera laminadas encoladas fueron manufacturadas por la única industria de este ramo, hasta el presente en Venezuela, la cual se encuentra localizada en la Zona Industrial de Matanzas, de la Ciudad de Puerto Ordaz, Estado Bolívar.

La madera empleada para la fabricación de las vigas laminadas fue a partir de fustes completos de pino caribe provenientes de las plantaciones del sur del estado Monagas. Y como se dijo anteriormente estas plantaciones se encuentran bajo el manejo de la empresa del Estado CVG-Proforca, la cual esta encargada de plantar, explotar y realizar estudios de mejoramiento genético de esta especie para asegurar la buena adaptación y poder obtener los mejores resultados de la misma, y es de recalcar que estas son las únicas plantaciones de pino caribe de gran escala manejadas sustentablemente por el estado en el país.

Longwood (1962), describe esta madera como moderadamente pesada, generalmente con olor resinoso fuerte pero sin sabor distintivo, con grano generalmente recto, duramen color marrón rojizo, el cual varía con la cantidad de resinas, la albura es de color pálido y anillos de crecimiento claramente definidos por bandas de tejido denso.

Posteriormente esta madera fue procesada en el Aserradero San Luís de la Corporación Forestal Uverito, para ser transformados en láminas, según espesores, definidos en la Tabla 1. El adhesivo empleado fue Metil-di-isocianato (MDI), identificado comercialmente bajo el nombre de Jowaton, y fabricado por la industria alemana Jowa.

Tabla 1. Identificación de las principales características técnicas de las vigas laminadas encoladas de pino caribe. Fuente Elaboración propia.

\begin{tabular}{|c|c|c|c|c|c|c|c|}
\hline \multirow{3}{*}{$\begin{array}{l}\text { Viga } \\
\mathrm{N}^{\circ}\end{array}$} & \multicolumn{7}{|c|}{ Características de las vigas } \\
\hline & \multirow{2}{*}{ Especie } & \multicolumn{3}{|c|}{ Medidas Promedios } & \multirow{2}{*}{$\begin{array}{c}\text { Tipo de laminación de la } \\
\text { Viga }\end{array}$} & \multirow{2}{*}{$\begin{array}{c}\text { Número de } \\
\text { láminas de } \\
\text { madera en la } \\
\text { sección } \\
\text { transversal }\end{array}$} & \multirow[b]{2}{*}{$\begin{array}{l}\text { Espesor promedio de las } \\
\text { láminas (mm) }\end{array}$} \\
\hline & & $\begin{array}{c}\text { Ancho } \\
(\mathrm{mm})\end{array}$ & $\begin{array}{l}\text { Altura } \\
(\mathrm{mm})\end{array}$ & $\begin{array}{l}\text { Longitud } \\
(\mathrm{mm})\end{array}$ & & & \\
\hline 1 & Pino caribe & 65 & 274 & 3.500 & Finger joint & 8,0 & 3,4 \\
\hline 4 & Pino caribe & 65 & 270 & 3.500 & Finger joint & 8,0 & 3,5 \\
\hline 5 & Pino caribe & 67 & 273 & 3.500 & Finger joint & 8,0 & 3,5 \\
\hline
\end{tabular}

\begin{tabular}{|l|l|l|l|l|l|l|l|}
\hline 2 & Pino caribe & 65 & 284 & 3.500 & Lámina Completa & 8,0 & 3.5 \\
\hline 3 & Pino caribe & 65 & 285 & 3.500 & Lámina Completa & 8,0 & 3,5 \\
\hline 6 & Pino caribe & 65 & 273 & 3.500 & Lámina Completa & 8,0 & 3,5 \\
\hline
\end{tabular}

\begin{tabular}{|l|l|l|l|l|l|l|l|}
\hline 7 & Pino caribe & 93 & $\mathbf{2 8 3}$ & 3.500 & Finger joint & 8,0 & 3,5 \\
\hline 8 & Pino caribe & 93 & $\mathbf{2 8 3}$ & 3.500 & Finger joint & 8,0 & 3,5 \\
\hline 9 & Pino caribe & 93 & 283 & 3.500 & Finger joint & 8,0 & 3,4 \\
\hline
\end{tabular}

\begin{tabular}{|l|l|l|l|l|l|l|l|}
\hline 10 & Pino caribe & 98 & 284 & 3.500 & Lámina Completa & 8,0 & 3,5 \\
\hline 11 & Pino caribe & 97 & 289 & 3.500 & Lámina Completa & 8,0 & 3,5 \\
\hline 12 & Pino caribe & 98 & 283 & 3.500 & Lámina Completa & 8,0 & 3,5 \\
\hline
\end{tabular}

\begin{tabular}{|l|l|l|l|l|l|l|l|}
\hline 14 & Pino caribe & 125 & 275 & 3.500 & Finger joint & 8,0 & 3,5 \\
\hline 17 & Pino caribe & 125 & 282 & 3.500 & Finger joint & 8,0 & 3,5 \\
\hline 18 & Pino caribe & 127 & 280 & 3.500 & Finger joint & 8,0 & 4,0 \\
\hline
\end{tabular}

\begin{tabular}{|l|l|l|l|l|l|l|l|}
\hline 13 & Pino caribe & 125 & 292 & 3.500 & Lámina Completa & 11,00 & 2.5 \\
\hline 15 & Pino caribe & 128 & $\mathbf{2 8 5}$ & 3.500 & Lámina Completa & 11,00 & 2,5 \\
\hline 16 & Pino caribe & 125 & $\mathbf{2 8 5}$ & 3.500 & Lámina Completa & 11,00 & 2,5 \\
\hline
\end{tabular}


Luego que la materia prima fue acondicionada se prepararon dos tipos de listones que iban a conformar la viga laminada. Un tipo de estos listones fue del largo total de la viga (sin divisiones o secciones), y el otro tipo fue seccionado en pequeños retazos tratando de eliminar la mayor cantidad de nudos y defectos de los listones largos originales, para lo cual, a estos retazos de madera se les practicó en los extremos la técnica del tipo unión dentada (finger joint), para así poderlos unirlos y conformar el listón definitivo. Luego que los listones, en ambos casos estuvieron listos se encolaron uno sobre otro por su sección más ancha y se prensaron en frío, elaborando así, las vigas laminadas encoladas rectas, y de sección rectangular en el plano transversal (Tabla 1),

Es importante destacar que en todas las fases de selección de la materia prima (rolas), selección de los listones, mecanizado (labrado mecanizado, finger joint, etc) y en todo el proceso de elaboración de las vigas laminadas el Laboratorio Nacional de Productos Forestales (LNPF) no intervino, puesto que era preciso saber en que forma estaba trabajando esta empresa y poder evaluar tanto el proceso de manufactura como el producto final.

\section{B. Sitio de desarrollo de la investigación.}

La investigación se desarrolló en la Sección de Ensayos, Laboratorio de Propiedades Físicas y Mecánicas de la Madera del Laboratorio Nacional de Productos Forestales (LNPF-ULA-MPPA) adscrito a la Facultad de Ciencias Forestales y Ambientales de la Universidad de Los Andes, Mérida, Venezuela. Una vez recibidas la totalidad de vigas en el laboratorio, se dispuso de forma inmediata a su identificación y registro de las principales características morfológicas de las vigas laminadas encoladas, las cuales se reportan detalladamente en la Tabla 1, Luego se procedió a introducirlas al cuarto de acondicionamiento para llevar el contenido de humedad de estas vigas aproximadamente a un $12 \%$, el cual es requerido por la norma.

\section{Procedimientos técnicos para determinar los esfuerzos de diseño de las vigas laminadas encoladas a escala natural a partir de los ensayos de flexión, según la Norma ASTM D-198-84.}

El presente trabajo de investigación consistió en ensayar a flexión las vigas laminadas encoladas según la norma (ver fig. 1), lo que permitió la determinación de los esfuerzos de diseño presentados en la Tabla 2.

El procedimiento para el ensayo de la propiedad mecánica de flexión estática realizado a cada una de las vigas laminadas de pino caribe encoladas con adhesivo de MDI fue someter a la viga, al momento del ensayo en flexión, a cargas simétricas perpendiculares a la dirección del grano, logrando someter esta porción de la viga a un momento flector uniforme libre de corte (Figura 1). Cada una de las vigas, fueron ensayadas a la velocidad de $2,5 \mathrm{~mm} / \mathrm{min}$. Paralelamente se registraron las observaciones coordinadas, por parte de los técnicos del Laboratorio de Ensayos e investigadores responsables del proyecto, de las cargas y deflexiones. Este procedimiento se realiza hasta que se produzca la ruptura o falla en la viga. Los equipos empleados para el desarrollo de este ensayo fueron los siguientes:

- Máquina de ensayo. RIEHLE - Prensa universal. Capacidad 60 toneladas.

- Aparatos de soporte (platos de apoyos o reacciones y soporte lateral).

- Aparato de carga. La carga se aplicó a través de bloques de madera sobre el ancho total de la sección de cada una de las vigas, siendo ésta de suficiente espesor para evitar la concentración de altos esfuerzos en los puntos de contacto entre la viga y el bloque de madera, asegurando un radio de curvatura, en la cara de contacto del bloque de madera con la viga, entre 2 a 4 veces la altura de la viga. La carga total fue aplicada por igual en dos puntos equidistantes de las reacciones. Los dos puntos de aplicación de la carga estaban a una distancia igual de $1 / 3$ de la luz (Figura 1). 
- Aparato para medir la deflexión (deflectómetro) del eje axial de la viga. Marca CSE con una precisión de $0,01 \mathrm{~mm}$.

Desde el punto de vista de procedimiento se siguieron cada una de las principales exigencias técnicas propuestas por la Norma ASTM D-198-84, en referencia a:

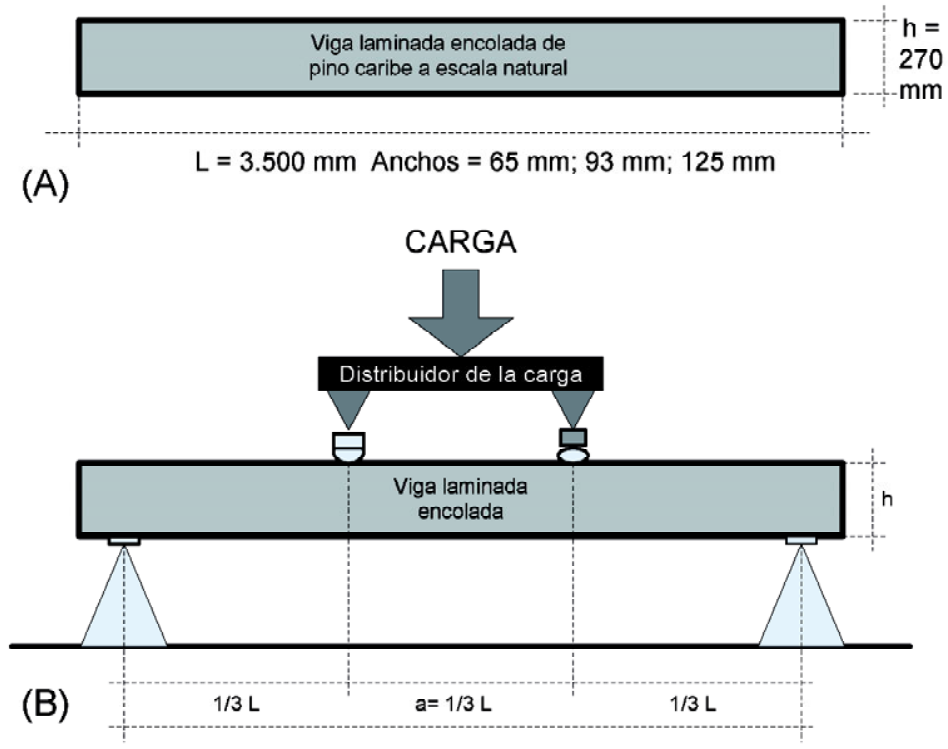

Figura 1. (A) Esquema dimensional de las vigas. (B) Esquema de la distribución de cargas sobre las vigas.

- Descripción técnica de las vigas de prueba.

Se usaron vigas de madera laminada de pino caribe encoladas con adhesivo de MDI manufacturadas en la ciudad de Puerto Ordaz. Al momento de la llegada de las vigas al LNPF-ULA-MPPA se procedió a registrar las dimensiones de las vigas y la obtención del promedio de las mismas, tal como lo exige la norma, para cada una de las muestras (Tabla 1). Parte de la medición como la longitud, el tipo y orientación de las fibras de las piezas de madera en cada una de las vigas se procedió a tomarlas con mayor detalle. Para el ensayo a flexión, las vigas tenían una relación de a/h que se ubica entre 5:1 y 12:1, es decir, la reacción entre la distancia y el punto de aplicación de la carga es relativamente larga, siendo según la norma la relación más deseada. De igual modo se realizó un diagnóstico técnico a fin de identificar la procedencia del material, especie, historia, características de procesamiento mecánico y de los tratamientos de conservación que pueden llegar a afectar la resistencia de la madera o de las líneas de cola, por lo que, una vez ubicadas las vigas en el Laboratorio de Ensayos del LNPF-ULAMPPA, se procedió al registro de las principales imperfecciones o de cualquier modificación intencional efectuadas sobre la composición física de las vigas (Figuras 2, 3 y 4) y para esto se desarrollaron planillas individuales indicando todas estas características, pudiendo observarse que los defectos mayormente presentes fueron: Mancha azul (68\% y 53\%), Nudos (20\% y 33\%), leño juvenil presente $(7 \%$ y $6 \%)$ y defectos de labrado $(2 \%$ y $4 \%)$ en las vigas conformadas por láminas unidas por finger joint y enteras respectivamente. 


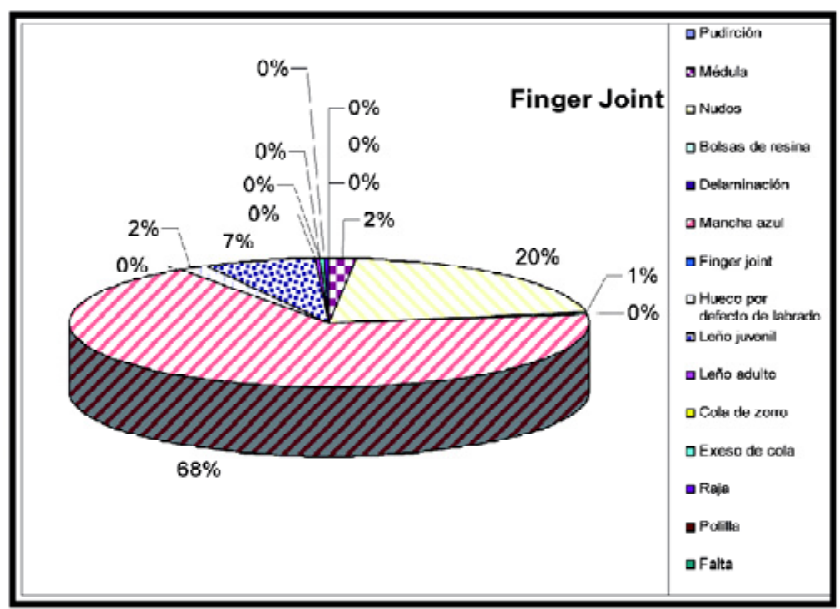

Figura 2. Porcentaje de defectos presentes en las vigas laminadas conformadas por láminas seccionadas y unidas por Finger Joint.

Fuente: Elaboración propia

\section{- Procedimiento:}

Una vez que las vigas alcanzaron el $\mathrm{CH} \% 12 \pm 2$ en el cuarto de acondicionamiento se procedió al desarrollo de los ensayos mecánicos. Se identificó el tamaño de cada sección, la luz, la distancia entre la reacción y el punto de aplicación de la carga de cada una de las muestras (Tabla 1). Las pruebas de flexión fueron desarrolladas a una velocidad constante alcanzando la carga máxima aproximadamente entre los 6 y 20 minutos. Posteriormente se anotaron cada uno de los datos de las cargas y de las deflexiones en la primera falla, en la carga máxima y en los puntos donde ocurrieron cambios repentinos. De igual modo, por cada ensayo de flexión se describieron los principales detalles de las fallas según su tipo, manera y orden de ocurrencia y en la posición en la viga. Una vez obtenidos los resultados finales de cada uno de los valores de las cargas máximas, se procedió al cálculo de los esfuerzos de diseño para flexión según lo planteado en el apéndice X2 de la norma. De este modo se pudo determinar los valores del Esfuerzo Límite Proporcional (ELP), Módulo de Ruptura (MOR), Módulo de Elasticidad (MOE) (Tabla 2).

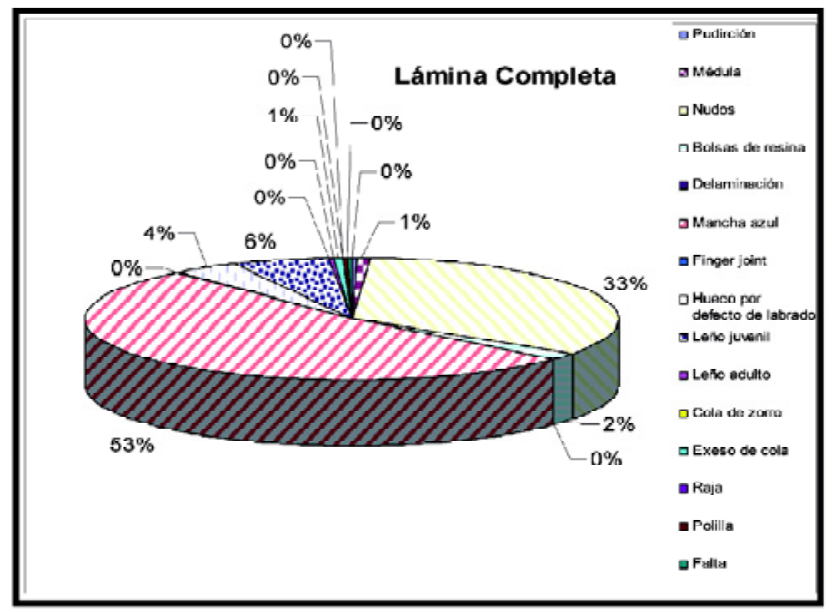

Figura 3. Porcentaje de defectos presentes en las vigas laminadas conformadas por láminas enteras. Fuente: Elaboración propia. 


\section{RESULTADOS Y DISCUSIÓN}

\section{A. Determinación de los esfuerzos de diseño de las vigas laminadas de pino caribe y adhesivo MDI.}

Previo al análisis de los resultados de los esfuerzos de diseño expuestos en las Tablas 2 y 3 , es importante resaltar la factibilidad de fabricación de elementos estructurales de pino caribe, a partir de la tecnología de la madera laminada encolada. A pesar de que en Venezuela existe poca bibliografía especializada, que exponga las verdaderas potencialidades de uso que tiene el pino caribe para fines estructurales dentro de la temática tratada. Estudios propuestos, entre otros, por Ninin (1994), Owen de Contreras y Contreras (1997), Contreras (2003), reivindican, con criterios de innovación y nuevos productos forestales para sistemas estructurales a partir esta especie maderable.

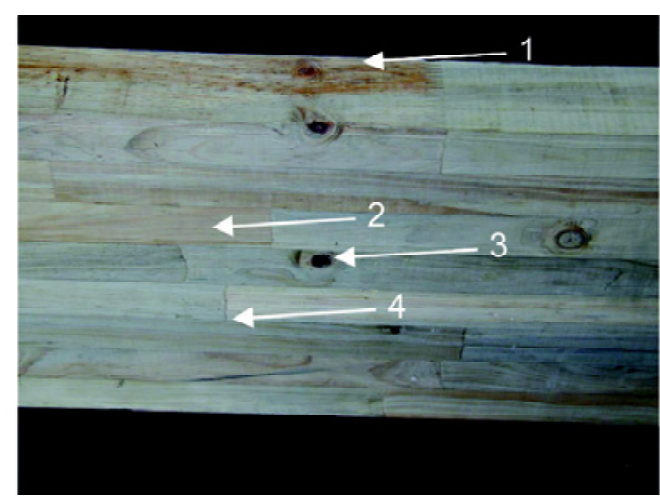

Figura 4. Detalle de una de las vigas, denotándose la presencia de depósitos de resina (1), leño juvenil (2), nudos (3) y finger joint (4). Foto: Mary Owen de Contreras

Es pertinente resaltar la visión y los notables esfuerzos financieros y tecnológicos de la empresa fabricante de las vigas objeto de estudio, primero, al apostar por una tecnología poco conocida en Venezuela dentro de la industria de la construcción, como lo es la madera laminada encolada.

Segundo, la experiencia de fabricación de elementos laminados de pino caribe era única para finales de los años noventa, época en la cual la industria se encontraba en fase de instalación y en la actualidad la fabricación de este tipo de elementos sigue siendo única. Eso sin contar, el cierto desconocimiento técnico en lo que se refería, a las características anatómicas de esta madera, especialmente a la influencia negativa de dos tipos diferentes de madera (leño juvenil y leño adulto), las cuales se encuentran dentro de un mismo fuste o rola; los tipos de adhesivos más idóneos para la fabricación exitosa de los elementos estructurales; el significado real de la técnica de uniones dentadas (finger joint); las estrategias de marketing para ubicar con éxito en el mercado nacional e internacional; los diferentes tipos de productos manufacturados y por manufacturar; los procesos intrínsecos referidos a lo que es un flujograma de procesos industriales de manufactura de productos de madera laminada; y otros. Esta labor ha sido titánica por parte del grupo inversor, hecho que dignifica su presencia como industria, en multitud de edificaciones en todo el territorio nacional donde están presentes sus productos forestales de madera laminada encolada.

Se puede apreciar en la Tabla 1, la fabricación de un total de 18 vigas. Dentro de este grupo las vigas presentaron notables diferencias en el diseño y fabricación de los productos. Además del defecto dimensional de las secciones transversales, las vigas también presentaron diferencias en la composición interna de las láminas de madera, específicamente en lo que se refería a vigas fabricadas donde se hace uso de la técnica de finger joint $\left(\mathrm{N}^{\mathrm{o}} 1,4,5,7,8,9,14,17,18\right)$. Razón por la cual se realizó un labrado 
mecanizado para uniformizar todas las vigas a las tres dimensiones finales en la sección transversal, con anchos de $65 \mathrm{~mm}, 95 \mathrm{~mm}$ y $125 \mathrm{~mm}$ (Tabla 3 ). El resto de vigas fueron realizadas con láminas de madera enteras (sin seccionar) del largo total del elemento estructural $\left(\mathrm{N}^{\circ} 2,3,6,10,11,12,13,15,16\right)$ (Tabla 2).

Tabla 2. Determinación de los esfuerzos de diseño a partir de la Norma ASTM D-198-84, de las vigas laminadas de pino caribe encoladas con adhesivo de MDI. Fuente Elaboración propia.

\begin{tabular}{|c|c|c|c|c|c|c|c|c|c|}
\hline $\begin{array}{c}\text { Viga } \\
\mathrm{N}^{\circ}\end{array}$ & $\begin{array}{c}\text { Especie de } \\
\text { madera }\end{array}$ & $\begin{array}{c}\text { Luz } \\
\text { entre } \\
\text { apoyos } \\
\text { (mm) }\end{array}$ & $\begin{array}{l}\text { Longitud } \\
\text { Eje de } \\
\text { Carga - } \\
\text { Punto de } \\
\text { Apoyo } \\
\text { (mm) }\end{array}$ & $\begin{array}{l}\text { Carga } \\
\text { Máxima } \\
\text { (lb) }\end{array}$ & $\begin{array}{c}\text { Carga al } \\
\text { Esfuerzo al } \\
\text { Límite } \\
\text { Proporcional } \\
\text { (lb) }\end{array}$ & $\begin{array}{l}\text { Deformación } \\
\text { (mm) }\end{array}$ & $\begin{array}{l}\text { Esfuerzo al } \\
\text { Límite } \\
\text { Proporciona } \\
\text { (ELP)1 } \\
\left(\mathrm{N} / \mathrm{mm}^{2}\right)\end{array}$ & $\begin{array}{l}\text { Modulo de } \\
\text { Ruptura } \\
\text { (MOR) } \\
\left(\mathrm{N} / \mathrm{mm}^{2}\right)\end{array}$ & $\begin{array}{c}\text { Módulo de } \\
\text { Elasticidad } \\
\text { (MOE) } \\
\left(\mathrm{N} / \mathrm{mm}^{2}\right)\end{array}$ \\
\hline 1 & Pino caribe-FJ & 2.500 & 845 & $8.820,00$ & 7.717 .50 & 270 & 17,80 & 20,34 & $3.186,76$ \\
\hline 4 & Pino caribe-FJ & 2.500 & 845 & $10.584,00$ & $7.938,00$ & 295 & 18,85 & 25,14 & $3.135,35$ \\
\hline 5 & Pino caribe-FJ & 2.500 & 845 & $13.230,00$ & $9.261,00$ & 374 & 20,87 & 29,82 & $2.707,85$ \\
\hline
\end{tabular}

\begin{tabular}{|l|l|l|l|l|l|l|l|l|l|}
\hline 2 & Pino caribe-EC & 2.500 & 845 & $14.553,00$ & $6.615,00$ & 166 & 14,20 & 31,24 & $3.989,83$ \\
\hline 3 & Pino caribe-EC & 2.500 & 845 & $12.568,50$ & $9.922,50$ & 236 & 21,15 & 26,79 & $4.165,46$ \\
\hline 6 & Pino caribe-EC & 2.500 & 845 & $11.907,00$ & $9.261,00$ & 309 & 21,52 & 43,03 & $3.378,31$ \\
\hline
\end{tabular}

\begin{tabular}{|l|l|l|l|l|l|l|l|l|l|}
\hline 7 & Pino caribe-FJ & $\mathbf{2 . 5 0 0}$ & 845 & $14.553,00$ & $10.584,00$ & 234 & 15.99 & 21,99 & $3.198,84$ \\
\hline 8 & Pino caribe-FJ & 2.500 & 845 & $13.781,25$ & $9.261,00$ & 218 & 13,99 & 20,83 & $3.004,42$ \\
\hline 9 & Pino caribe-FJ & 2.500 & 845 & $15.876,00$ & $11.907,00$ & 247 & 17,99 & 23,99 & $3.409,29$ \\
\hline
\end{tabular}

\begin{tabular}{|l|l|l|l|l|l|l|l|l|l|}
\hline 10 & Pino caribe-EC & 2.500 & 845 & $23.814,00$ & $11.907,00$ & 255 & 16,96 & 33,91 & $3.100,86$ \\
\hline 11 & Pino caribe-EC & 2.500 & 845 & $17.640,00$ & $13.296,00$ & 280 & 18,47 & 24,51 & $3.023,48$ \\
\hline 12 & Pino caribe-EC & 2.500 & 845 & $17.199,00$ & $15.876,00$ & 415 & 22,77 & 24,66 & $2.567,49$ \\
\hline
\end{tabular}

\begin{tabular}{|l|l|l|l|l|l|l|l|l|l|}
\hline 14 & Pino caribe-FJ & 2.500 & 845 & $18.522,00$ & $13.230,00$ & 192 & 15,75 & 22,05 & $3.951,41$ \\
\hline 17 & Pino caribe-FJ & 2.500 & 845 & $24.696,00$ & $14.553,00$ & 217 & 16,48 & 27,96 & $3.566,45$ \\
\hline 18 & Pino caribe-FJ & 2.500 & 845 & $19.404,00$ & $13.230,00$ & 210 & 14,96 & 21,93 & $3.368,71$ \\
\hline & & & & & & & & & \\
\hline 13 & Pino caribe-EC & 2.500 & 845 & $25.357,50$ & $16.537,50$ & 284 & 17,46 & 26,78 & $2.789,29$ \\
\hline 15 & Pino caribe-EC & 2.500 & 845 & $17.529,75$ & $14.553,00$ & 273 & 15.75 & 18,98 & $2.681,92$ \\
\hline 16 & Pino caribe-EC & 2.500 & 845 & $11.907,00$ & $9.261,00$ & 176 & 10,27 & 13,20 & $2.710,83$ \\
\hline
\end{tabular}

$\mathrm{FJ}=$ Viga laminada conformada por láminas de madera seccionadas con finger joint . $\mathrm{EC}=$ Viga laminada conformada por láminas de madera completa.

El análisis de la Tabla 3 expone que no existió una uniformidad en los resultados de los esfuerzos de diseño como lo son el Esfuerzo Límite Proporcional (ELP), Módulo de Ruptura (MOR) y Módulo de Elasticidad (MOE). Resaltando que los valores más altos pertenecen a las vigas laminadas cuyas láminas no fueron seccionadas por la técnica del finger joint (EC). 
Tabla 3. Resultados promedios por ancho de viga y tipo (Finger joint/lámina completa) de los esfuerzos de diseño a partir de la Norma ASTM D-198-84, de las vigas laminadas de pino caribe encoladas con adhesivo de MDI. Fuente Elaboración propia.

\begin{tabular}{|c|c|c|c|c|c|c|c|}
\hline \multirow[b]{2}{*}{ Especie } & \multirow[b]{2}{*}{$\begin{array}{l}\text { Tipo de unión } \\
\text { de las láminas } \\
\text { en las vigas }\end{array}$} & \multirow[b]{2}{*}{$\begin{array}{c}\text { Ancho } \\
\text { nominal de } \\
\text { Viga (mm) }\end{array}$} & \multirow[b]{2}{*}{$L$} & \multirow[b]{2}{*}{$\begin{array}{l}\text { Cantidad de } \\
\text { Vigas }\end{array}$} & \multicolumn{3}{|c|}{ Valores Promedios } \\
\hline & & & & & $\begin{array}{l}\text { Esfuerzo Límite } \\
\text { Proporcional } \\
(\text { ELP)(N/mm²) }\end{array}$ & $\begin{array}{c}\text { Módulo de } \\
\text { Ruptura (MOR) } \\
\left(\mathrm{N} / \mathrm{mm}^{2}\right)\end{array}$ & $\begin{array}{c}\text { Módulo de } \\
\text { Elasticidad } \\
\text { (MOE) } \\
\left(\mathrm{N} / \mathrm{mm}^{2}\right)\end{array}$ \\
\hline $\begin{array}{l}\text { Pino } \\
\text { caribe }\end{array}$ & Finger joint & 65 & 3.500 & 3 & 19,178 & 25,101 & $3.009,985$ \\
\hline $\begin{array}{l}\text { Pino } \\
\text { caribe }\end{array}$ & $\begin{array}{l}\text { Lámina } \\
\text { completa }\end{array}$ & 65 & 3.500 & 3 & 18,958 & $33,689\left(^{*}\right)$ & $3.844,532\left(^{*}\right)$ \\
\hline $\begin{array}{l}\text { Pino } \\
\text { caribe }\end{array}$ & Finger joint & 95 & 3.500 & 3 & 15,994 & 22,269 & $3.204,186$ \\
\hline $\begin{array}{l}\text { Pino } \\
\text { caribe }\end{array}$ & $\begin{array}{l}\text { Lámina } \\
\text { completa }\end{array}$ & 95 & 3.500 & 3 & $19,398\left(^{*}\right)$ & 27,693 & $2.897,270$ \\
\hline $\begin{array}{l}\text { Pino } \\
\text { caribe }\end{array}$ & Finger joint & 125 & 3.500 & 3 & 15,729 & 23,984 & $3.628,858$ \\
\hline $\begin{array}{l}\text { Pino } \\
\text { caribe }\end{array}$ & $\begin{array}{l}\text { Lámina } \\
\text { completa }\end{array}$ & 125 & 3.500 & 3 & 14,495 & 19,651 & $2.727,348$ \\
\hline
\end{tabular}

*Valores más altos.

Los mejores valores corresponden a las vigas constituidas por láminas completas. El mejor valor de ELP corresponde a la viga con ancho de $95 \mathrm{~mm}$, y los mejores valores de MOR y MOE corresponden a las vigas con ancho de $65 \mathrm{~mm}$. Esto pudiera inferir técnicamente que estas vigas son las mejores, en razón de que la tecnología de finger joint usada en la fabricación de las otras vigas fue aplicada de manera excesiva, y en vez de ocurrir lo que expresaba Freas y Selbo (1954), que esta técnica permitía eliminar los defectos más significativos de la madera en procura de la mejora de las propiedades mecánicas de un elemento estructural, lo que hicieron fue debilitarlas como se muestra en los resultados de la tabla 3. Pudiendo ser la causante de estos bajos valores la cantidad de nudos presentes y defectos de fabricación en ambas vigas como se mostró en las figuras 1, 2 y 3.

\section{B. Análisis comparativo del procedimiento de cálculo de los esfuerzos de diseño a partir de los ensayos de flexión estática, según la Norma ASTM D-198-84 y la Norma del JUNAC (1983), PAT- REFORT de vigas de madera a escala natural.}

Siguiendo las recomendaciones expuestas en la Norma ASTM D-198-84, se logró calcular los esfuerzos de diseño (ELP, MOR, MOE), a partir de los ensayos de flexión estática para las vigas laminadas (Tablas 2 y 3). Se debe reconocer que en el contexto de los Países Andinos, que involucra a Venezuela, Colombia, Ecuador, Bolivia y Perú, la Junta de Acuerdo de Cartagena (JUNAC), por medio de toda una serie de normas y manuales (PADT-REFORT), han sido excelentes guías, desde inicio de la década de los años ochenta, para el desarrollo, estandarización y promoción en todo lo referente a la ciencia, tecnología y uso de la madera y los productos forestales.

De ahí que en la Tabla 4, y dada la importancia antes planteada de la JUNAC, se exponga una comparación en los procedimientos de cálculo de los esfuerzos de diseño obtenidos a partir de la norma ASTM D-198-84 y la PAT-REFORT de la JUNAC (1982) de ensayos de vigas de madera a escala natural. La comparación denota que los valores determinados por la norma ASTM, es más rigurosa en el procedimiento de cálculo, razón por la cual los valores son más bajos. Al comparar los valores obtenidos de los esfuerzos de diseño de las vigas laminadas encoladas de pino caribe, arrojó valores más altos a partir del uso de las formulas propuestas por la norma JUNAC (1983) (ELP 35,108 $\mathrm{N} / \mathrm{mm}^{2}$; MOR 40,123 N/mm²; MOE 6.310,634 N/mm²) respecto a los valores obtenidos por la norma ASTM, incrementando en casi un $50 \%$ los valores de la esta última. 
Tabla 4. Comparación de los resultados de la determinación de esfuerzos de diseño para una viga laminada encolada de pino caribe, entre la Norma ASTM D-198-84

y la JUNAC PAT- REFORT (1982). Fuente Elaboración propia.

\begin{tabular}{|c|c|c|c|}
\hline \multirow{2}{*}{ Propiedad } & \multicolumn{2}{|c|}{ Métodos } & \multirow{2}{*}{$\begin{array}{c}\text { Diferencia cuantitativa en casi } \\
\text { un 50\% más, entre ambos } \\
\text { métodos en N/mm }\end{array}$} \\
\cline { 2 - 3 } & $\begin{array}{c}\text { Normas ASTM } \\
\left(\mathrm{N} / \mathrm{mm}^{2}\right)\end{array}$ & JUNAC $\left(\mathrm{N} / \mathrm{mm}^{2}\right)$ & 17.308 \\
\hline $\begin{array}{c}\text { Esfuerzo al Límite } \\
\text { Proporcional (ELP). }\end{array}$ & 17.800 & 35.108 & 19,780 \\
\hline Módulo de Ruptura (MOR) & 20,343 & 40,123 & $3.123,875$ \\
\hline $\begin{array}{c}\text { Módulo de Elasticidad } \\
\text { (MOE) }\end{array}$ & $3.186,759$ & $6.310,634$ & \\
\hline
\end{tabular}

\section{Análisis de las características anatómicas macroscópicas (morfológicas) de las vigas laminadas y su influencia en la determinación de los valores finales de esfuerzos de diseño.}

El análisis técnico de las características morfológicas de los elementos estructurales, se basó en el análisis visual de cada una de las dieciocho (18) vigas manufacturadas. Se ha podido percibir, en esta primera etapa de consolidación de la industria, que las vigas laminadas encoladas para aumentar la calidad de sus esfuerzos de diseño, deben ser mejoradas en lo que respecta a los siguientes aspectos técnicos: proceso de selección y clasificación de la madera, según la madera de leño juvenil y leño adulto; eliminación de los verticilos de nudos, pudrición, bolsas de resina, grietas y aristas faltantes; y mejora de la calidad del proceso de aserrado y labrado mecanizado, especialmente en el buen uso de la técnica de finger joint, para la eliminación de los defectos naturales en las láminas de madera. Finalmente, con los resultados obtenidos en el presente trabajo, y considerando el contexto técnico anterior, se espera que las vigas laminadas de pino caribe encoladas con adhesivo Metil-di-isocianato (MDI), puedan ser empleadas de manera confiable y de distintas formas con fines estructurales, específicamente como vigas y viguetas (Figura 5). 

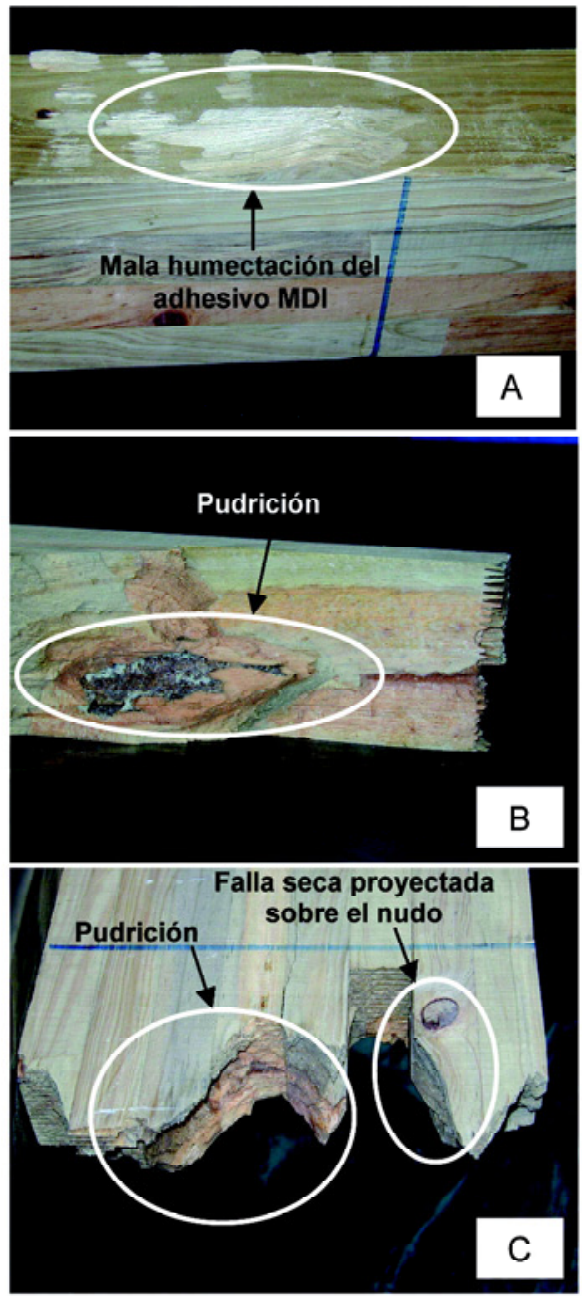

Figura 5. Proyección gráfica que identifica algunos de los defectos técnicos encontrados en las vigas. (A) Mala calidad de la línea de cola. (B) Pudrición en las láminas de madera. (C) Efecto de los defectos de pudrición y nudos en la zona de falla de la viga. Fotos: Mary Owen de Contreras.

\section{Comparación de los esfuerzos de diseño, de las vigas estudiadas, con otros elementos estructurales de madera aserrada y vigas laminadas.}

A fin de validar la calidad estructural de las vigas manufacturadas, se procedió a comparar los valores obtenidos de los esfuerzos de diseño de las vigas de pino caribe con otros trabajos similares. Esto permite inferir, en esta primera etapa, si las vigas laminadas encoladas pueden ser usadas de forma confiable en diferentes usos con fines estructurales.

Se procede a comparar los resultados promedios obtenidos en la presente investigación (Tablas 2 y 3), con los valores correspondientes a la madera sólida aserrada de pino caribe, según los grupos de madera con calidad estructural, propuestos Centeno (1983), éstos se ubican ligeramente por debajo de los valores de $\operatorname{MOE}\left(7,832 \mathrm{~N} / \mathrm{mm}^{2}\right)$ del Grupo C $\left(0,32<\right.$ Densidad $\left.<0,39 \mathrm{~g} / \mathrm{cm}^{3}\right)$. 
Posteriormente se comparan los esfuerzos de diseño de las vigas de madera laminada encolada de pino caribe con las vigas de pino radiata de Chile y el pino resinoso de Argentina, la Tabla 5 expone que los valores de las vigas estudiadas (Tabla 2 y 3 ), están muy por debajo de los valores comparados.

Todo este contexto permite inferir que las vigas estudiadas, bajo los principios técnicos antes analizados y que involucran la presente investigación, al ser colocada a mayores exigencias de uso estructural en cualquier edificación, pudiera poner en riesgo la estabilidad, rigidez y seguridad de la misma, especialmente a un momento de una mayor solicitud en la resistencia estructural, caso de un sismo. De ahí que se recomienda, en la segunda fase de consolidación y desarrollo de los procesos de manufactura y diseño de productos de la industria de madera laminada evaluada, considerar la mejora continua de cada uno de los puntos reportados en el presente trabajo, para la comercialización exitosa y segura en el tiempo de sus productos en el ámbito nacional e internacional.

Tabla 5. Comparación de los resultados promedios de los esfuerzos de diseño de las vigas laminadas encoladas de madera de pino caribe, manufacturadas con finger joint, respecto a las vigas laminadas encoladas de pino radiata (Pinus radiata) de Chile expuestos por la Cámara Chilena de la Construcción (2007), y el pino resinoso (Pinus Elliottii) de Argentina determinados por Astori y Natalini (2002). Fuente Elaboración propia.

\begin{tabular}{|l|c|c|c|c|}
\hline \multirow{2}{*}{ Especie } & Tipo de unión de las & \multicolumn{3}{|c|}{ Valores Promedios } \\
\cline { 3 - 5 } & láminas en las vigas & $\begin{array}{c}\text { Esfuerzo al Límite } \\
\text { Proporcional-ELP } \\
\left(\mathrm{N} / \mathrm{mm}^{2}\right)\end{array}$ & $\begin{array}{c}\text { Modulo de Ruptura- } \\
\mathrm{MOR} \\
\left(\mathrm{N} / \mathrm{mm}^{2}\right)\end{array}$ & $\begin{array}{c}\text { Módulo de } \\
\text { Elasticidad - MOE } \\
\left(\mathrm{N} / \mathrm{mm}^{2}\right)\end{array}$ \\
\hline Pino caribe & Finger joint & $19,178^{*}$ & $25,101^{*}$ & $3.009,985^{*}$ \\
\hline $\begin{array}{l}\text { Pino } \\
\text { radiata }\end{array}$ & Finget joint & $\mathrm{X}$ & $\mathrm{X}$ & $\begin{array}{c}\text { Grado A } \\
(11.000,000) \\
\text { Grado B }( \\
9.000,000)\end{array}$ \\
\hline $\begin{array}{l}\text { Pino } \\
\text { resinoso }\end{array}$ & Finger joint & $\mathrm{X}$ & $\mathrm{X}$ & $8.238,000$ \\
\hline
\end{tabular}

- (*) Valores reportados para las vigas de finger joint de ancho $6500 \mathrm{~mm}$.

- $\mathrm{X}=$ Valores no reportados en el ensayo.

- $1 \mathrm{~N} / \mathrm{mm}^{2}=1 \mathrm{MPa}$

\section{CONCLUSIONES Y RECOMENDACIONES}

El desarrollo de la presente investigación permitió determinar los esfuerzos de diseño de las vigas laminadas encoladas con calidad estructural, de madera de pino caribe y adhesivo MDI. Las mismas fueron manufacturadas por la única industria de madera laminada establecida en Venezuela y localizada en la ciudad de Puerto Ordaz, estado Bolívar. La evaluación técnica se desarrolló en el Laboratorio Nacional de Productos Forestales (LNPF-ULA-MPPA), Mérida, Venezuela. Los elementos evaluados corresponden a la primera etapa de consolidación de la industria, es decir, a la fase del proceso de instalación, mejora de procesos industriales, y diseño de productos laminados. Se ensayaron la totalidad de 18 vigas, clasificadas en tres tipos. Todas iguales en altura $(270 \mathrm{~mm}$.) y longitud (3500 mm.), pero diferentes en sus dimensiones de ancho (65 mm.; $95 \mathrm{~mm}$.; $125 \mathrm{~mm}$.), así como en la composición del número de láminas vistas en la sección transversal, uso de láminas completas de madera, y las realizadas con el uso de la tecnología de uniones dentadas (finger joint). 
Al comparar los valores obtenidos de los esfuerzos de diseño de las vigas laminadas encoladas de pino caribe, determinadas a partir del uso de las fórmulas propuestas por la Norma ASTM D-198-84 respecto a la Norma JUNAC (1982), los valores fueron más bajos en casi un 50\%. Además, al comparar los resultados promedios obtenidos en la presente investigación, con los valores correspondientes a la madera sólida aserrada de pino caribe, según los grupos de madera con calidad estructural, propuestos Centeno (1983), éstos se ubican muy por debajo de los valores de $\operatorname{MOE}\left(7,832 \mathrm{~N} / \mathrm{mm}^{2}\right)$ perteneciente al Grupo estructural C (Densidad 0,39>D $<0,32 \mathrm{~g} / \mathrm{cm}^{3}$ ). De igual forma sucedió al comparar los bajos valores obtenidos de esfuerzos de diseño de las vigas estudiadas, con los valores de los esfuerzos de diseño de madera laminada encolada de pino radiata de Chile y pino resinoso de Argentina.

Todo ello permite inferir, en esta primera etapa de consolidación de la industria, que las vigas laminadas encoladas, para aumentar la calidad de sus esfuerzos de diseño, deben ser mejoradas en lo que respecta a los siguientes aspectos técnicos: proceso de selección y clasificación de la madera según la madera de leño juvenil y leño adulto; eliminación de los verticilos de nudos, pudrición, resina, grietas y aristas faltantes; y mejora de la calidad del proceso de aserrado y labrado mecanizado, especialmente en el buen uso de la técnica de finger joint. Finalmente, con los resultados obtenidos en el presente trabajo, y considerando el contexto técnico anterior, se espera que las vigas laminadas de pino caribe y adhesivo Metil-di-isocianato (MDI), deben ser rediseñadas tanto en la fase de selección y clasificación de la materia prima, procesos de manufactura y diseño de los productos, a fin de que puedan ser empleadas de forma confiable en diferentes usos con fines estructurales, específicamente como vigas y viguetas.

\section{BIBLIOGRAFÍA}

Astori, R.; Natalini, M. 2002. Uso estructural del pino resinoso (Pinus Elliottii) en vigas de madera laminada. p. 14. Facultad de Ingeniería. Universidad Nacional de Nordestes. Argentina.

Cámara Chilena de la Construcción. (En Línea). Esfuerzos de diseño del pino radiata. ((Fecha de consulta 10 de Marzo 2007). Disponible en: http://www.registrocdt.cl/

Centeno, J. 1983. Normas para la Clasificación de Madera Estructural. p. 45, Instituto Forestal Latinoamericano (IFLA). Mérida, Venezuela, 1983.

Contreras, W. 2003. Tres prototipos de viviendas de bajo costo con madera y acero para el medio rural venezolano. p. 145, UFORGA- ULA. CEFAP-ULA. Universidad de Los Andes. Mérida, Venezuela.

CVG Proforca. (En Línea). Inversiones. (Fecha de consulta 13 de Marzo 2007). Disponible en: http://www.cvgproforca.com

Freas A.D.; Selbo, M.L. 1954. Fabrication and design of glued laminated wood. Structural members. P. 346. Forest Products Laboratory. Wisconsin. USA. 1954.

JUNAC. 1982. Manual de diseño y normas para ensayos de madera. p. 175, Junta del Acuerdo de Cartagena (JUNAC). Lima, Perú.

LONGWOOD, F. 1962. Commercial Timbers of the Caribbean. U.S. Department of Agriculture. Handbook 207. Washington, D.C. 
Ninin, L. 1995. Necesidades de un esfuerzo de adecuación de técnicas y equipos de aserrado para el producto de nuestras plantaciones de crecimiento rápido. Instituto Forestal Latinoamericano (IFLA). Mérida, Venezuela 17(2):56 - 74.

Owen de C. M.; Contreras, W. 1997. Elaboración de un elemento laminado, tipo Parallam, con tiras de pino caribe Pinus caribaea Var. Hondurensis y resina fenol - formaldehído. Rev. For. Vzolana 41(2): 129-136. 